University of Wollongong

Research Online

Faculty of Social Sciences - Papers (Archive) Faculty of Arts, Social Sciences \& Humanities

$1-1-2020$

\title{
A Matched Control Trial of a Mental Health Literacy Intervention for Parents in Community Sports Clubs
}

Diarmuid Hurley

University of Wollongong, dsh725@uowmail.edu.au

Mark S. Allen

University of Wollongong, markal@uow.edu.au

Christian Swann

Stewart A. Vella

University of Wollongong, stvella@uow.edu.au

Follow this and additional works at: https://ro.uow.edu.au/sspapers

Part of the Education Commons, and the Social and Behavioral Sciences Commons

Research Online is the open access institutional repository for the University of Wollongong. For further information contact the UOW Library: research-pubs@uow.edu.au 


\title{
A Matched Control Trial of a Mental Health Literacy Intervention for Parents in Community Sports Clubs
}

\author{
Abstract \\ 2020, Springer Science+Business Media, LLC, part of Springer Nature. This controlled trial evaluated the \\ effectiveness of a mental health literacy intervention for parents delivered through community sport \\ clubs. In total, 540 parents ( 321 females, 219 males) of adolescent athletes participated in a brief \\ educational workshop on youth mental health $(n=352)$ or a community-matched control group $(n=188)$. \\ Generalised linear mixed models revealed no significant improvements in the intervention group \\ compared to control in the primary mental health literacy outcomes, at 1 month follow-up. However, \\ parents in the intervention group were more likely to seek formal help for themselves, had increased \\ confidence and knowledge to help someone experiencing a mental health disorder, experienced reduced \\ psychological distress, and perceived more support from other parents in their sport club, relative to the \\ control group. Overall, the findings suggest that a brief educational intervention delivered through \\ community sports clubs can positively affect some components of parents' mental health literacy. \\ Disciplines \\ Education | Social and Behavioral Sciences

\section{Publication Details} \\ Hurley, D., Allen, M., Swann, C. \& Vella, S. (2020). A Matched Control Trial of a Mental Health Literacy \\ Intervention for Parents in Community Sports Clubs. Child Psychiatry and Human Development,
}


A matched control trial of a mental health literacy intervention for parents in community sports clubs 


\begin{abstract}
This controlled trial evaluated the effectiveness of a mental health literacy intervention for parents delivered through community sport clubs. In total, 540 parents (321 females, 219 males) of adolescent athletes participated in a brief educational workshop on youth mental health $(n=352)$ or a community-matched control group $(n=188)$. Generalised linear mixed models revealed no significant improvements in the intervention group compared to control in the primary mental health literacy outcomes, at one month follow-up. However, parents in the intervention group were more likely to seek formal help for themselves, had increased confidence and knowledge to help someone experiencing a mental health disorder, experienced reduced psychological distress, and perceived more support from other parents in their sport club, relative to the control group. Overall, the findings suggest that a brief educational intervention delivered through community sports clubs can positively affect some components of parents' mental health literacy.
\end{abstract}

Keywords: brief intervention; child and adolescent mental health; prevention 
A matched control trial of a mental health literacy intervention for parents in community sports clubs

\section{Introduction}

1

2

3

4

5

6

7

8

9

Mental health disorders are estimated to affect approximately $13.4 \%$ of children and adolescents worldwide, constituting a significant threat to the health and well-being of young people (1). Reported help-seeking rates vary but remain low with only $18-34 \%$ of adolescents with a mental health problem seeking professional help (2), and less than a quarter of young children accessing treatment services for mental health issues (3). In Australia, a large scale national survey found that $13.9 \%$ of children and adolescents aged 4-17 years were assessed as having a mental health disorder but almost one third of those did not seek professional help (4). Young people have a strong preference to seek out informal sources of support such as family and friends (5), but are more likely to engage in help-seeking behaviours and receive professional help if encouraged and supported by their family (6).

Parents in particular play a key role in the help-seeking process for young people with mental health disorders (7). A recent study identified 78 distinct roles and behaviours that parents engage in to support their child's mental health, such as facilitating treatment and maintaining a positive outlook (8). Previous research has identified the need to target parents and the family system in order to improve child and adolescent mental health outcomes (9, 10). For example, meta-analyses of parenting programs for the prevention of child and adolescent mental health disorders found beneficial outcomes, such as a reduction in mental health disorders, substance abuse and risky behaviours, from 11 years (10), up to 20 years post-intervention (11). However, the impact of interventions explicitly targeting parent and caregiver mental health literacy has received limited research attention.

Mental health literacy refers to knowledge, attitudes, beliefs, and help-seeking practices which aid in the prevention, identification, treatment and management of mental 
health disorders $(12,13)$. Research suggests that the mental health literacy of parents and caregivers is limited in terms of their confidence and ability to assist adolescents who experience a mental health disorder $(14,15)$. Inadequate mental health literacy in parents and caregivers can have adverse consequences for child and adolescent mental health outcomes including missed symptoms, delayed or non-engagement with youth mental health services, and higher levels of stigma $(4,16,17)$. Indeed, a recent systematic review found that prominent barriers to accessing youth mental health services included limitations in parents' awareness, knowledge and understanding of youth mental health disorders and services, as well as concerns about perceived stigma (18). Parental mental health literacy is also influenced by ethnic, cultural and religious group membership, values and belief systems (19), with poor understanding of mental health literacy and limited accessibility to mental health treatment services in non-Western countries (20). Higher levels of mental health literacy have been found in White, higher educated, and female populations (7). Mental health literacy can be targeted at the individual, community and population levels (13). Interventions are designed to increase participants' mental health awareness and knowledge, reduce stigma, encourage help-seeking and engagement with mental health services, and facilitate the development of skills and confidence to effectively help and support others (21). Importantly, mental health literacy interventions are assumed to result in a transfer of beneficial outcomes from those participating to others over time, such as a parent encouraging or getting help for their child (21). Interventions have targeted parental mental health literacy in the workplace (22), and in community settings $(23,24,25)$ resulting in improvements in parent's knowledge of, and confidence in dealing with, mental health disorders and treatment. One intervention included a 2-week online psychoeducational program for parents in the workplace and provided information on depression and anxiety in youth, help-seeking services and supportive actions parents can take (22). Another study 
involved a brief, face-to-face intervention including educational seminars on youth mental health and small discussion groups of parents (23). Qualitative evaluation indicated that parents perceived an increase in their knowledge of mental health disorders and treatments, and increased confidence to handle youth mental health issues. Two further studies targeted youth-referent mental health literacy of adult gatekeepers (including parents) in community samples $(24,25)$. In both studies, gatekeepers improved their mental health literacy from preto post-intervention. However, findings from these studies are somewhat limited by the lack of a control group or longitudinal follow-up.

Community sport clubs represent a potentially fruitful avenue for mental health promotion and the prevention of mental health disorders. Approximately half of all children and adolescents participate in organized sport worldwide, with higher rates of participation in developed countries (26). Access to a large community participation base coupled with the perceived health benefits of youth sport participation make sport clubs an ideal setting to engage in health promotion $(27,28)$. However, few sport organizations actively engage in health promotion or mental health initiatives, especially at the grassroots community level (29). In community sport clubs, parents often fulfil the role of team coach, manager and other volunteer positions, and adolescent sport participation is predominantly facilitated by parents. Furthermore, parents represent a key source of support for their child's sport participation (30). Parents of young athletes have reported that they attempt to buffer and mirror their child's emotions related to sport participation and use sport as a means to enhance or improve the parent-child relationship, facilitate communication and feel emotionally connected with their child $(31,32)$. Therefore, youth sport clubs appear an ideal setting to engage with parents and build on their role as a support provider. Indeed, findings from multiple qualitative studies demonstrate the acceptability of intervening with parents through community sport clubs $(15,33,34)$. In addition, these studies indicate the importance of 
parent social networks within community sport clubs for parenting support and advice. Two studies have targeted parents and other adult leaders in a community sport club context demonstrating improvements in participants' youth-referent mental health literacy $(35,36)$. However, both trials were uncontrolled and time intensive. For example, the "Read the Play" intervention required 8 hours of participation in educational seminars over three consecutive weeks (35).

The purpose of this study was to develop and evaluate the effectiveness of a mental health literacy intervention workshop delivered through community sport clubs. Based on findings from a pilot intervention (33), it was hypothesised that compared to parents in the matched control group, participants in the intervention group would show increases in the primary outcomes of mental health literacy. Primary outcomes were those that directly addressed mental health literacy (12,13), and included knowledge of the symptoms of depression and anxiety, knowledge of and attitudes towards mental health and help-seeking, confidence to seek help and communicate about mental health, and help-seeking intentions. Secondary outcomes related to the broader effect of the intervention on parents, but which were not directly targeted by the intervention, include parental psychological distress and perceived support from other parents within the sport club. Qualitative findings related to participants' perceptions of the program, including an evaluation of program content, design, reach, and engagement are discussed elsewhere (34).

\section{Participants}

\section{Method}

In total, 540 parents $\left(M_{\text {age }}=47.42 \pm 5.33\right.$ years $)$, comprised of 321 mothers $(59.4 \%)$ and 219 fathers, agreed to participate in the study. Parents were recruited from sport clubs in two regions of New South Wales, Australia. All parents who had an adolescent participating in youth sport within these communities were eligible to participate. Neighborhood socioeconomic position was determined according to the Socio-Economic Indexes for Areas 
Index of Relative Socio-Economic Disadvantage [SEIFA] (37) using parents' home postcodes. Nationwide, SEIFA scores are calculated to have a mean of 1000 and SD of 100 . Participants in the current study ranged from $788\left(2^{\text {nd }}\right.$ percentile $)-1125\left(100^{\text {th }}\right.$ percentile $)$ with a mean of $1011.41\left(\mathrm{SD}=51.58 ; 58^{\text {th }}\right.$ percentile $)$. Ten parents identified as Aboriginal or Torres Strait Islander. Thirteen parents spoke a language other than English at home.

\section{Procedure}

Following ethical approval from an institutional research ethics committee and in partnership with regional and national sporting bodies, youth sports clubs in the Illawarra and South Coast/South East Sydney, and Central Coast regions of Australia were contacted via telephone and email and invited to participate. Initial contact was made through the club president/committee members. Following approval, the intervention was the offered to all parents of adolescent athletes within participating clubs and organisations. Multiple recruitment strategies were used, including advertisements (with permission) on sport clubs' social media and website pages and on a regional sporting body's website. The first author and members of the research team also visited youth sport clubs on training and match days at the start of the season to increase visibility, develop trust, and facilitate recruitment. Interested parents voluntarily provided their contact details to receive further information about the study (via phone or email). Participants were recruited and workshops conducted over the course of three sport seasons (two winter sport seasons and one summer sport season). Examples of winter sports include Football and Australian Rules Football while an example of a summer sport is Cricket.

In total, 18 workshops were delivered, with an average of 20 parents $(\mathrm{SD}=24.85)$.

The majority of workshops took place at local sport club facilities while two workshops took place at other community venues and seven at a university campus. Participant numbers in each workshop ranged from two in a small regional club to 103 from a large urban sport 
academy. All participants provided written informed consent. Participants in the intervention group completed study measures prior to taking part in the intervention workshop. The duration of the workshops varied from $50-75$ minutes ( $M=62$ minutes) depending on group size, parent engagement and discussion. Workshops were led by the first author who had mental health first aid certification, experience in delivering mental health workshops, and working in sport club settings. Following the workshop, parents received a pamphlet containing key information from the workshop and were also directed to the Ahead of the Game project website (https://aheadofthegame.org.au/) and other online resources should they require or request more information.

\section{Measures}

Anxiety literacy and depression literacy. The Anxiety Literacy questionnaire (ALIT; 5) and the Depression Literacy questionnaire (D-LIT; 38) contain 22 statements measuring disorder specific knowledge and attitudes. In both questionnaires nine statements that did not correspond to intervention aims and content were excluded. Participants responded to 13 statements about depression and anxiety respectively with response categories of 1 (true), 2 (false), or 3 (I don't know). An example statement is: "People with depression/anxiety often speak in a rambling or disjointed way". One point is given for a correct response. Higher scores are indicative of better anxiety or depression literacy. Both the A-LIT and D-LIT have demonstrated adequate construct validity and test-retest reliability in adult samples (5). In the current study both depression literacy ( $\alpha=.73)$, and anxiety literacy $(\alpha=.74)$ demonstrated adequate internal reliability.

Mental health literacy. Mental health literacy was assessed using an adapted 16-item version of the Mental Health Literacy scale (39) which examines knowledge of, and attitudes toward, mental health and help seeking. The scale is comprised of three subscales. The first subscale contains two items measuring knowledge and confidence to seek information and 
communicate about mental health $(\alpha=.71)$. An example statement is: "I am confident that I know where to seek information about mental illness." The second subscale contains nine items measuring attitudes that promote recognition of mental illness or appropriate helpseeking behavior $(\alpha=.82)$. An example statement is: "If I had a mental illness I would not tell anyone." The third subscale contains five items evaluating stigmatizing attitudes $(\alpha=$ .89). An example statement is: "How willing would you be to make friends with someone with a mental illness." Items are rated on a 5-point scale from 1 (strongly disagree) to 5 (strongly agree). The scale is appropriate to identify those with low levels of mental health literacy as well as changes in mental health literacy resulting from an intervention program (39). The Mental Health Literacy scale has been found to have adequate test-retest reliability and construct validity in adult samples (39).

General help seeking. The general help seeking questionnaire (GHSQ) is a widely used measure of help seeking intentions associated with future help seeking behavior (40). Participants are asked to rate the likelihood of seeking help from four personal (e.g., friend) and four professional (e.g., general practitioner) sources on a scale from 1 (extremely unlikely) to 7 (extremely likely). The GHSQ has demonstrated acceptable predictive and construct validity and test-retest reliability (42). In this study participants were asked to separately rate the likelihood of using four personal $(\alpha=.95)$ and four professional $(\alpha=.89)$ help-seeking options for themselves, as well as the likelihood of seeking help for their adolescent from personal sources $(\alpha=.90)$ or professional sources $(\alpha=.95)$. measure of their overall confidence to help someone experiencing a mental health problem, developed for the purpose of the study, from 1 (not at all confident) to 5 (extremely confident): "If you had contact with someone who appeared to be experiencing a mental 173 health problem, how confident would you feel in helping them." 
Parent social support networks in the sport club environment. Two statements

were developed for the purpose of the study to investigate the nature of the relationship and support between parents in the sport club environment $(\alpha=.87)$. Parents were asked to rate the extent to which they agree or disagree on a scale of 1 (strongly disagree) to 5 (strongly agree) to the statements "I feel supported by other parents in my sport club" and "I have other parents I can talk to in my sport club if I'm having troubles."

Parent psychological distress. The Kessler-6 (K6; 41) is a six-item short screening measure of psychological distress. Participants are asked to rate how often they have felt, for example, "restless or fidgety" in the past 30 days from 1 (none of the time) to 5 (all of the time). The $\mathrm{K} 6$ has good internal consistency and test-retest reliability (current study, $\alpha=.81$ ) and is suitable for use with different demographic adult samples (41).

\section{Design}

The intervention was developed based on the identified mental health literacy needs of parents of youth athletes (15) and findings from a pilot intervention with parents delivered through community sport clubs (33). The intervention was also one component of a wider mental health promotion and prevention intervention series for parents, coaches and adolescents offered through community sport clubs (Ahead of the Game; 42), and employing a community based participatory approach (CBPR; 43). In the intervention group, 352 parents (195 women, 157 men; $M_{\text {age }}=47.70 \pm 5.14$ years $)$ participated in a 1 hour intervention workshop. A control group, who completed study measures at two time points but received no intervention, was made up of 188 parents ( 126 women, 62 men; $M_{\text {age }}=46.96$ \pm 5.63 ) from a matched community. The control region was selected based on population size, number of adolescent sport participants, socioeconomic position, and sport culture, relative to the intervention region. 
Intervention development. In formative research, parents identified a need for clear,

basic, brief information (that fit within their schedule) on youth mental health and the actions

they can take to support and assist their adolescents (15). The aim of the intervention

workshop was to increase parent mental health literacy by educating parents on symptoms of depression and anxiety disorders in adolescents, mental health resources and treatment options, and communicating about mental health (42). Moreover, the workshop was set out to increase parents' confidence and preparedness to provide support to their adolescents and others experiencing mental health problems. The intervention was designed to maximize reach and engagement of parents and overcome common barriers to participation such as time constraints, inconvenience, inaccessibility and stigma $(18,44)$. This was achieved by being brief, convenient, available to all parents, and supported by key stakeholders in the community sport club. Previous studies also revealed the importance of social links between parents in sport clubs and offering opportunities for interaction and discussion $(15,33)$. increased use of videos, and discussion of scenarios relating to normal or problematic teenage behavior. A brief (approximately 1 hour) in-person workshop, supplemented by print and online content was developed. Intervention content was guided by the mental health literacy framework (12), and was designed to be engaging through a mix of parent reflection, discussion, and presentation (see Table 1). The content focused on improving knowledge by

217 identifying symptoms of depression and anxiety, two of the most prevalent mental health 218 disorders in children and adolescents, and informing parents of the various professional and other help-seeking resources available to them. Furthermore, commonly held misconceptions or negative perceptions of those with mental health disorders were challenged. In addition, parents were guided on how to communicate about mental health with their adolescent in order to improve confidence and intentions to provide help. Based on feedback from the pilot 
intervention, discussion of the reality of the help-seeking process and the difficulties parents may encounter was added.

Materials were developed and adapted in accordance with Mental Health First Aid guidelines $(45,46)$, or used with permission from mental health organizations and parenting organizations (e.g., ReachOut, Raising Children Network). The content of the intervention workshop was assessed for relevance and accuracy by a Mental Health First Aid trainer. Parents' ability to distinguish between regular teenage behavior and the signs of a possible mental health disorder was highlighted as a particularly important intervention component that warranted more attention. Therefore, it was decided to incorporate additional content, such as the use of scenarios for parents to evaluate and discuss as a group, depicting either "typical" teenage behavior or symptoms indicative of a mental health disorder.

\section{Data Analyses}

Independent samples $t$-tests were used to examine baseline differences between the intervention and control groups, and to examine any differences between dropouts and participants who completed follow-up measures. To establish the effectiveness of the intervention, between-group mean differences on the primary and secondary outcomes were analyzed using linear mixed models (LMM) in IBM SPSS 25.0, with clustering at club-level and team-level included as a random intercept. The Group (intervention and control), Time (baseline and one month follow-up), and Group x Time interaction were the main fixed effects with parent sex, personal experience of mental health issues, and previous experience of contact with others with a mental health disorder, age and socioeconomic status included as covariates. These variables were selected as covariates on account of their previously established associations with mental health literacy $(7,47)$. Main effects of the interaction term adjusted for covariates are reported. In addition, significant Group by Time interactions were investigated further through moderation analysis to identify potential moderator effects 
of parent sex, personal diagnosis of a mental health disorder, and previous contact with someone with a mental health disorder.

The linear mixed model can be used to test for between-group effects while also incorporating the multilevel structure of the data as a random effect. This approach is also suitable for dealing with missing data using restricted maximum likelihood estimation and dealing with unequal sample sizes across groups (48). A sensitivity analysis was also conducted excluding all participants with missing data to check on the robustness of results. We report findings from the model with imputed missing data and report sensitivity analyses where findings differ. Model assumptions of homoscedasticity and normal distribution of data were met.

\section{Results}

Baseline measures were completed by 540 parents. Of these, 352 parents were in the intervention group and 188 were in the control group. Follow-up measures were completed by 284 parents $(52.6 \%)$ at one month, with 226 parents $(64.2 \%)$ in the intervention group and 58 parents $(30.9 \%)$ in the control group. Forty-nine parents $(9.1 \%)$ over the total sample had personally experienced a diagnosed mental health problem in the last three months. More than two in five parents $(43.1 \%)$ had contact with someone who had a mental health problem in the last three months with $92.7 \%$ of these parents attempting to provide help. Participants in the intervention group $(M=1015.42)$ had lower levels of socioeconomic disadvantage than those in the control group $(M=1003.830), t(537)=3.53, p<.001, d=.24$. Attrition analyses showed that compared to those who returned, dropouts had higher levels of stigmatizing attitudes, $t(516)=2.43, p=.015, d=.21$, greater confidence to seek information and communicate about mental health, $t(534)=1.98, p=.048, d=.17$, and higher levels of social disadvantage, $t(534)=1.98, p<.001, d=.35$. 
Mean scores, significance values, and confidence intervals for all measured outcomes

274

across intervention and control groups at baseline and at one-month follow-up are presented in Table 2. In terms of primary outcomes, there was a significant between-group difference for parents' likelihood of seeking professional help. Compared to parents in the control group, parents in the intervention group increased their likelihood of seeking professional help from pre- to post-intervention, $F(1,324.15)=5.43, p=.020, \eta_{\mathrm{p}}^{2}=.016$. Compared to parents in the control group, parents in the intervention group also increased their confidence to seek information and communicate about mental health from pre- to post-intervention, $F(1$, $362.74)=12.91, p<.001, \eta_{\mathrm{p}}{ }^{2}=.034$. Follow-up analyses revealed that significant Group $\mathrm{x}$ Time interactions were not moderated by parent sex, personal diagnosis of a mental health disorder, or previous contact with someone with a mental health disorder (see Table 4). There were no significant between-group differences for attitudes about mental health, $F(1,317.77)=2.27, p=.133, \eta_{\mathrm{p}}^{2}=.007$, or stigmatizing attitudes, $F(1,307.85)=$ $0.96, p=.327, \eta_{\mathrm{p}}^{2}=.003$. There were also no significant between-group differences for depression literacy, $F(1,336.73)=0.89, p=.345, \eta_{\mathrm{p}}{ }^{2}=.002$, anxiety literacy $F(1,325.86)=$ $2.00, p=.129, \eta_{\mathrm{p}}^{2}=.006$, informal help-seeking, $F(1,344.83)=0.14, p=.709, \eta_{\mathrm{p}}^{2}=.000$, formal help seeking for child, $F(1,355.14)=1.44, p=.231, \eta_{\mathrm{p}}^{2}=.004$ or informal helpseeking for child, $F(1,420.94)=0.11, p=.740, \eta_{\mathrm{p}}^{2}=.000$. A complete case sensitivity analysis was conducted with statistical tests re-run with only those participants with complete data, with no meaningful change to results.

There were significant between-group differences in secondary outcomes including parents' psychological distress, $F(1,348.03)=5.62, p=.018, \eta_{\mathrm{p}}^{2}=.015$, such that, compared to parents in the control group, parents in the intervention group decreased in their levels of psychological distress from pre- to post-intervention. Follow-up analyses revealed parents' decrease in psychological distress in the intervention group was moderated by personal 
mental health disorder diagnosis, $F(1,304.77)=5.28, p=.022, \eta_{\mathrm{p}}{ }^{2}=.017$, such that only those with a personal mental health disorder showed a significant improvement in psychological distress. Compared to parents in the control group, parents in the intervention group also increased their perceived support from other parents in the sport club, $F(1,332.41)$ $=4.52, p=.034, \eta_{\mathrm{p}}^{2}=.013$, and confidence to address a mental health issue, $F(1,356.41)=$ $4.21, p=.041, \eta_{\mathrm{p}}{ }^{2}=.011$, from pre- to post-intervention. Follow-up analyses revealed that Group x Time interactions were not moderated by parent sex or previous contact with someone with a mental health disorder (see Table 4). Statistical tests were re-run for participants with complete data, with no meaningful change to results.

Although between-group differences were not found on some of the primary outcomes, there was a significant main effect for Time on depression literacy, $F(1,335.26)=$ $19.60, p<.001, \eta_{\mathrm{p}}^{2}=.055$, such that depression-related knowledge increased from preintervention to one-month follow-up in both groups. There was also a significant increase in anxiety literacy, $F(1,324.49)=16.76, p<.001, \eta_{\mathrm{p}}{ }^{2}=.049$, knowledge and confidence to seek information and communicate about mental health, $F(1,360.68)=5.45, p=.020, \eta_{\mathrm{p}}{ }^{2}=$ .014 , and likelihood to seek formal help, $F(1,322.56)=11.61, p<.001, \eta_{\mathrm{p}}^{2}=.035$, from preto post-intervention. There was also a significant decrease in stigmatizing attitudes, $F(1$, $306.32)=6.53, p=.011, \eta_{\mathrm{p}}^{2}=.020$, and likelihood to seek informal help for their child, $F(1$, $418)=82.41, p<.001, \eta_{\mathrm{p}}^{2}=.160$. There were no significant main effects for Group indicating that participants in experimental and control groups did not differ on any primary or secondary outcomes.

In examining those factors associated with mental health literacy, it was found in both groups that previous contact with someone who had a mental health disorder was related to higher levels of depression literacy, $F(1,431.29)=22.06, p<.001, \eta_{\mathrm{p}}{ }^{2}=.049$, anxiety literacy, $F(1,432.52)=15.37, p<.001, \eta_{\mathrm{p}}^{2}=.034$, knowledge and confidence to seek 
information and communicate about mental health, $F(1,447.98)=18.66, p<.001, \eta_{\mathrm{p}}^{2}=.040$, confidence, $F(1,448.85)=9.27, p=.002, \eta_{\mathrm{p}}^{2}=.020$, positive attitudes towards mental health and help-seeking, $F(1,130.67)=15.53, p<.001, \eta_{\mathrm{p}}{ }^{2}=.106$, and lower stigmatizing attitudes, $F(1,452.02)=23.04, p<.001, \eta_{\mathrm{p}}{ }^{2}=.048$ (see Table 3 for means, standard deviations and effect sizes for all outcome variables ). Parent sex was also related to depression literacy, $F(1$, $427.95)=7.61, p=.006, \eta_{\mathrm{p}}{ }^{2}=.017$, such that females had greater knowledge of depression than males. Compared to males, females also had better anxiety literacy, $F(1,429.58)=$ $17.13, p<.001, \eta_{\mathrm{p}}{ }^{2}=.038$, greater knowledge and confidence to seek information and communicate about mental health, $F(1,459.65)=22.15, p<.001, \eta_{\mathrm{p}}^{2}=.046$, less stigmatizing attitudes, $F(1,457.88)=10.97, p<.001, \eta_{\mathrm{p}}^{2}=.023$, and more positive attitudes toward mental health, $F(1,435.69)=21.63, p<.001, \eta_{\mathrm{p}}{ }^{2}=.047$. Diagnosis of a personal mental health disorder was also related to psychological distress, $F(1,429.14)=80.14, p<$ $.001, \eta_{\mathrm{p}}{ }^{2}=.157$, such that those with a diagnosed disorder experienced more psychological distress.

\section{Discussion}

The aim of this experiment was to evaluate the effectiveness of a parent mental health literacy intervention delivered through community sports clubs. Parents in the intervention group improved their confidence and knowledge to assist someone experiencing a mental health disorder, and were more likely to seek out formal sources of help, demonstrating partial support for our hypothesis. However, parental depression and anxiety literacy, intentions to seek help for their adolescent, and attitudes towards mental health and helpseeking did not significantly improve in the intervention group compared to the control group, in contrast to the hypothesis. Rather, increases in anxiety and depression literacy, formal help-seeking intentions, and stigmatizing attitudes were observed in both intervention and control groups at one-month post-intervention. Additional findings revealed that parents 
in the intervention group perceived more support from other parents in their sport club, and experienced reduced psychological distress to a greater extent than those in a matched control condition.

An important finding from this research is that parents in the intervention group did not improve their mental health (depression and anxiety) literacy to a greater extent than those in the control condition. This is in contrast to findings from a recent qualitative evaluation of the current intervention whereby participants reported increased awareness and knowledge of the signs and symptoms of mental health disorders (34). Notably, improvements in mental health literacy were observed in both intervention and control groups. Improvements in the mental health literacy of control group participants might be explained by visible in club promotion and the completion of the in-depth mental health literacy questionnaire, despite not being involved in any formal intervention. These events might have triggered parents in the control group to research and discuss mental health related topics, and improve their base of knowledge. Indeed, measurement reactivity refers to the potential for an increase in participants' awareness and sensitivity to a particular variable under investigation following exposure to its measurement (49). This suggests that programs that increase parents' self-awareness might be sufficient to improve mental health literacy in parents. It is unclear, however, if parents in either intervention or control groups were exposed to any mental health related information or education in the month following the intervention. Exposure to such information represents a potential confounding factor and merits further investigation.

After completing the intervention, parents were more likely to seek out formal sources of help for themselves, and were more confident in knowing where to seek help compared to a control group, but this was not reflected in any increased willingness to seek out formal sources of support for their adolescent. Mental health literacy interventions assume a transfer 
of benefits (e.g., transfer of knowledge or helping behaviors) from those involved in the intervention to others in their family and social networks (21). That no change was observed in parents' help-seeking intentions for their adolescent might be explained by parents in both intervention and control groups rating themselves as likely to seek formal help for their adolescent at baseline, indicating ceiling effects. Indeed, parents in the qualitative evaluation also expressed their likelihood and preparedness to seek help for their adolescent, and reported that they used the intervention as a reference to discuss mental health with their adolescent (34). In addition, the help-seeking measures in this study did not account for the severity of the disorder or parental recognition of the disorder. This is important as previous research suggests that parents are more likely to seek help if they are able to recognize the symptoms of a mental health problem and if they are presented with more severe or confronting symptoms of a mental health disorder $(4,50)$.

One month after the intervention, parents in both groups rated themselves as more likely to seek help from formal sources (such as a GP) but were no more likely to seek help from informal sources. This finding is inconsistent with previous research on parents' preferences to seek help from informal sources $(5,51)$, and might be explained by an increase in parents' perceptions of the seriousness of mental health issues (13), and the emphasis within the intervention on seeking professional help when necessary. In addition, parents scored high on attitudes to promote and facilitate mental health treatment at baseline (and at follow-up) and this might explain why no change was observed in these attitudinal outcome measures in the intervention or control group (again suggesting ceiling effects). Our findings did however show that parents in the intervention group improved their confidence and knowledge to seek information and communicate about mental health, to a greater extent than those in the control group. This is an important finding as a lack of knowledge of helpseeking options is a prominent barrier to parents accessing support for their child $(4,14)$. 
This finding also supports those from the qualitative evaluation of the intervention, with parents perceiving that they had increased their knowledge, confidence and preparedness to communicate and assist someone experiencing a mental health issue (34).

In terms of secondary outcomes, parents in the intervention group indicated that they felt more support from their peers in the sport club after one-month (compared to control group participants). Past research indicates the importance of parent social support networks in sport clubs $(15,33,34)$. Friends and others in parent social networks represent a primary source of information, support and advice about mental health (14). Therefore, within the sport club environment, the ability of parents to both provide and make use of appropriate support and advice from other parents appears crucial when facing mental health related issues. Indeed, qualitative findings indicated that following the intervention, parents used information and resources provided in the intervention workshop to help and advise other parents in their social networks (34).

Findings from this study reveal that an intervention designed to improve parents' awareness, knowledge, and attitudes (referent to youth mental health) can reduce personal psychological distress in those with a diagnosed mental health disorder. This finding is consistent with a recent study that found parents reported less stress and fewer depressive symptoms three months after a family-based intervention for adolescent depression (52). How mental health literacy affects psychological distress is still somewhat unclear. Similar to the current study, a review of web-based interventions for the general public found that increases in mental health literacy were associated with reduced psychological distress, though the sample also included those without mental health disorders (53). In contrast, a large scale study of the prevalence of depression in Australian communities found that limited or poorer mental health literacy was associated with a reduced likelihood of a depression diagnosis (54). Further research is required to establish the link between mental 
health literacy (and its various components) and psychological well-being in different populations.

\section{Strengths and Limitations}

Strengths of this matched controlled trial included the use of a community based participatory research approach (43) in the process of recruiting and engaging sport clubs and parents. This approach necessitated the investment of time and resource into contacting clubs, building relationships with club members, and organizing workshops and survey completion with parents. Such an approach was taken to build awareness, trust and relationships in sport communities. As a result, a large sample of parents was recruited for the intervention.

Furthermore, this study used the mental health literacy framework $(12,13)$, and findings from formative (15) and pilot (33) research to target and evaluate parent mental health literacy across knowledge, attitudes and help-seeking components.

Despite these research strengths, there are a number of potential shortcomings that readers must consider in order to place the findings in context. First, due to the length and structure of the different sport seasons (winter and summer sports), and the organization of community sport clubs (mostly volunteer based), follow-up for long-term change in mental health literacy was not feasible, and it was necessary to limit follow-up to one month post intervention. Therefore, whether improvements are maintained long-term is unknown.

Completion of immediate post-intervention measures was also deemed infeasible as workshops were designed to be brief and were required to fit within parent and sport club schedules. Second, retention of participants was low (particularly in the control group) and some other form of incentive might be required in order to reduce attrition. However, even when incentives are offered dropout in mental health interventions for parents can be as high as $60 \%$ (55). Future research might seek to examine the social and organizational culture of 
447

different sports and sports clubs in understanding the potential effectiveness of a mental health literacy intervention.

A further potential limitation is that the sample was not fully representative of parents of adolescents. The intervention was offered to all parents of adolescent athletes within participating clubs with parents self-selecting into both the intervention and control groups. Parents with an interest in taking part due to recent experience with mental health issues (reported by at least $43 \%$ of participants) or a proactive approach to mental health education might have been overrepresented in the sample. It would also have been beneficial to assess how many parents had direct previous experience with mental health issues or treatment with their adolescents and children, as this could influence their decision to participate in a mental health intervention. In addition, mothers and female caregivers constituted $60 \%$ of the sample. Females tend to be more mental health literate than males $(7,56)$, as was observed in this study's findings. Intervention promotion and content might need to be targeted to specific individuals to encourage engagement for fathers and male caregivers and increase the representativeness of the sample.

In addition, children's continued participation in sport has been found to be predicted by higher parental education, higher household income, and English as the primary language spoken at home (57). In this study, only $2.4 \%$ of parents spoke a language other than English at home. However, as found in the current study, there was a large range of relative socioeconomic disadvantage reported among parents in community sport clubs with the mean SEIFA score falling very close to the population mean. Notwithstanding, the intervention could be offered to parents in other community parent and youth groups to enhance reach and engagement.

\section{Future Research}


Longer follow-up periods are needed to examine if improvements in mental health

472 literacy are maintained, and to further understand the effect of these improvements on child and adolescent mental health outcomes over time. Indeed, a recent meta-analysis of mental

474 health first aid interventions in adults found improvements in mental health literacy at six 475 months post-intervention but not at 12 months post-intervention (58). Research should also include a measure to account for participants' direct exposure (e.g., seeking out information) or indirect exposure (e.g., public health campaigns) to other mental health related education and information within the measurement period.

Future research is also warranted to investigate the potential mechanisms through which increased mental health literacy can influence psychological well-being. For example, one component of mental health literacy that was reinforced throughout the workshop was an emphasis on how to maintain positive well-being and self-care whilst also being able to support and assist others. Future research could also consider the potential added value of involving both parents and carers (where applicable) in the intervention. Only 16 parent/carer dyads were identified with both father and mother (or partners) of the same adolescent attending the intervention workshop

Further interventions might include additional content to provide more in-depth coverage of issues relating to youth mental health to build on participants' knowledge and confidence base with the intention of maximizing learning outcomes. Indeed, in the qualitative evaluation of the intervention, parents suggested diversifying intervention content and delivery channels as well as offering follow-up information post intervention (34). Such an approach might also aid in recruitment and engagement of parents. It could also be determined if multiple sessions lead to greater subsequent improvements in mental health literacy. The current workshop could also be evaluated in comparison to more intensive youth mental health programs such as the 14-hour youth mental health first aid course for adults 
who care for adolescents (YMHFA, 24). Similarly, further research could investigate how workshop dynamics (e.g., group size) might influence participants' experiences and learning outcomes, given the large variation in workshop participant numbers reported in the current study. For example, previous research has shown that smaller groups can facilitate increased parent engagement, reflection and retention of information, and can minimize parent concerns about disclosure and stigma (59).

\section{Summary}

This controlled trial evaluated the effectiveness of a brief mental health literacy intervention for parents delivered through community sport clubs. The design and development of the intervention was informed by the use of community based participatory research $(15,43)$. In sum, there were no significant improvements found in the primary outcomes of depression and anxiety literacy, mental health attitudes, or likelihood of seeking help for their adolescent, in the intervention group compared to control at one month followup. However, parents in the intervention group were more likely to seek formal help for themselves, had increased confidence and knowledge to help someone experiencing a mental health disorder, experienced reduced psychological distress, and perceived more support from other parents in their sport club, relative to the control group.

The current findings provide evidence that a brief single-session intervention can positively affect some components of parents' mental health literacy. However, given that the intervention group did not increase some aspects of mental health literacy to a greater extent than the control group, further refinement of components of the intervention might be required (that might incorporate additional sessions or content) in order to maximize benefits to parents. This study highlights the potential benefits and challenges in targeting interventions for parents' mental health literacy through the novel setting of community youth sport clubs. The findings of this experiment should be of interest to clinicians working 
521 with parents of children with mental health problems, and those designing community and

522 sport based interventions to reduce risk of mental health problems in children and

523 adolescents. We recommend further experimental studies that investigate long-term changes

524 in mental health literacy over time, and examine the potential transfer of benefit from parents

525 to adolescents, their families and wider community networks.

526

527 
528 Compliance with Ethical Standards

529 Conflicts of Interest: The authors declare no conflicts of interest.

530 Funding: This work was supported by funding from the Movember Foundation-The Mental

531 Health Imitative Grant 2014.

532 The funding source had no other involvement in the study design or completion.

533 Ethical approval: All procedures performed in studies involving human participants were in

534 accordance with the ethical standards of the institutional research committee and with the

5351964 Helsinki declaration and its later amendments or comparable ethical standards.

536 Informed consent: Informed consent was obtained from all individual participants included in

537 the study.

538 


\section{References}

540

541

542

543

1. Polanczyk GV, Salum GA, Sugaya LS, Caye A, Rohde LA (2015). Annual Research Review: A meta-analysis of the worldwide prevalence of mental disorders in children and adolescents. Journal of Child Psychology and Psychiatry 56:345-365.

2. Gulliver A, Griffiths KM, Christensen H (2010). Perceived barriers and facilitators to mental health help-seeking in young people: A systematic review. BioMed Central Psychiatry 10:113-122.

3. Oh E, Mathers M, Hiscock H, Wake M, Bayer J. (2015). Professional help seeking for young children with mental health problems. Australian Journal of Psychology 67:187-195.

4. Lawrence, D., Johnson, S., Hafekost, J., Boterhoven De Haan, K., Sawyer, M., Ainley, J., Zubrick SR (2015). The Mental Health of Children and Adolescents. Report on the second Australian Child and Adolescent Survey of Mental Health and Wellbeing. Canberra, Australia, Department of Health.

5. Gulliver A, Griffiths KM, Christensen H, Brewer JL (2012). A systematic review of help-seeking interventions for depression, anxiety and general psychological distress. BioMed Central Psychiatry 12:81-92.

6. Rickwood DJ, Deane FP, Wilson CJ (2007). When and how do young people seek professional help for mental health problems. Medical Journal of Australia 187:S3539.

7. Mendenhall AN, Frauenholtz S (2015). Predictors of mental health literacy among parents of youth diagnosed with mood disorders. Child and Family Social Work 20:300-309.

8. Honey A, Chesterman S, Hancock N, Llewellyn G, Hazell P, Clarke S (2015). Knowing what to do and being able to do it: Influences on parent choice and use of 
practices to support young people living with mental illness. Community Mental Health Journal 51:841-851.

9. Dardas LA, van de Water B, Simmons LA (2018). Parental involvement in adolescent depression interventions: A systematic review of randomized clinical trials. International Journal of Mental Health Nursing 27:555-570.

10. Yap MBH, Morgan AJ, Cairns K, Jorm AF, Hetrick SE, Merry S (2016). Parents in prevention: A meta-analysis of randomized controlled trials of parenting interventions to prevent internalizing problems in children from birth to age 18. Clinical Psychology Review 50:138-158.

11. Sandler IN, Schoenfelder EN, Wolchik SA, MacKinnon DP (2011). Long-term impact of prevention programs to promote effective parenting: Lasting effects but uncertain processes. Annual Review of Psychology 62:299-329.

12. Jorm AF, Korten, AE, Jacomb PA, Christensen H, Rodgers B, Pollitt P (1997). Mental health literacy: A survey of the public's ability to recognise mental disorders and their beliefs about the effectiveness of treatment. Medical Journal of Australia 166:182-186.

13. Jorm AF (2012). Mental health literacy: Empowering the community to take action for better mental health. American Psychologist 67:231-243.

14. Frauenholtz S, Conrad-Hiebner A, Mendenhall AN (2015). Children's mental health providers' perceptions of mental health literacy among parents and caregivers. Journal of Family Social Work 18:40-56.

15. Hurley D, Swann C, Allen MS, Okely AD, Vella SA (2017). The role of community sports clubs in adolescent mental health: The perspectives of adolescent males' parents. Qualitative Research in Sport, Exercise and Health 9:372-388. 
16. Gronholm PC, Ford T, Roberts RE, Thornicroft G, Laurens KR, Evans-Lacko S (2015). Mental health service use by young people: The role of caregiver characteristics. PloS One 10:e0120004.

17. Mendenhall A (2012). Predictors of Service Utilization Among Youth Diagnosed with Mood Disorders. Journal of Child and Family Studies 21:603-611.

18. Reardon T, Harvey K, Baranowska M, O’Brien D, Smith L, Creswell C (2017). What do parents perceive are the barriers and facilitators to accessing psychological treatment for mental health problems in children and adolescents? A systematic review of qualitative and quantitative studies. European Child Adolescent Psychiatry 26:623-647.

19. Hurley D, Swann C, Allen MS, Ferguson HL, \& Vella SA (2020). A systematic review of parent and caregiver mental health literacy. Community Mental Health Journal 56: 2-21.

20. Furnham A \& Hamid A (2014). Mental health literacy in non-western countries: A review of the recent literature. Mental Health Review Journal 19: 84-98.

21. Anderson RJ, Pierce D (2012). Assumptions associated with mental health literacy training: Insights from initiatives in rural Australia. Advances in Mental Health $10: 258-267$.

22. Deitz DK, Cook, RF, Billings DW, Hendrickson A (2009). Brief report: A web-based mental health program: Reaching parents at work. Journal of Paediatric Psychology $35: 488-494$.

23. Gilbo C, Knight T, Lewis AJ, Toumbourou JW, Bertino MD (2015). A qualitative evaluation of an intervention for parents of adolescents with mental disorders: The parenting challenging adolescents seminar. Journal of Child and Family Studies 24: $2532-2543$. 
24. Kelly CM, Mithen JM, Fischer JA, Kitchener BA, Jorm AF, Lowe A, Scanlan C (2011). Youth mental health first aid: A description of the program and an initial evaluation. International Journal of Mental Health Systems 5:4-12.

25. Story CR, Kirkwood AD, Parker S, Weller, BE (2016). Evaluation of the Better Todays/Better Tomorrows Youth Suicide Prevention Program: Increasing mental health literacy in rural communities. Best Practices in Mental Health 12:14-25.

26. Aubert S, Barnes JD, Abdeta C, Abi Nader P, Adeniyi AF, Aguilar-Farias N, ... \& Chang (2018). Global matrix 3.0 physical activity report card grades for children and youth: results and analysis from 49 countries. Journal of Physical Activity and Health 15(Supplement 2): S251-S273.

27. Geidne S, Quennerstedt M, Eriksson C (2013). The youth sports club as a healthpromoting setting: An integrative review of research. Scandinavian Journal of Public Health 41:269-283.

28. Kokko S (2014). Sports clubs as settings for health promotion: Fundamentals and an overview to research. Scandinavian Journal of Public Health 42:60-65.

29. Liddle SK, Deane FP, Vella SA (2016). Addressing mental health through sport: A review of sporting organizations' websites. Early Intervention in Psychiatry 11:93103.

30. Harwood CG, Knight CJ (2015). Parenting in youth sport: A position paper on parenting expertise. Psychology of Sport and Exercise 16:24-35.

31. Dorsch TE, Smith AL, McDonough MH (2009). Parents' perceptions of child-toparent socialization in organized youth sport. Journal of Sport Exercise Psychology 31: 444-468.

32. Stefansen K, Smette I, Strandbu Å (2018). Understanding the increase in parents' involvement in organized youth sports. Sport, Education and Society 23:162-172. 
33. Hurley D, Allen MS, Swann C, Okely AD, Vella SA (2018). The development, pilot, and process evaluation of a parent mental health literacy intervention through community sports clubs. Journal of Child and Family Studies 27:2149-2160.

34. Hurley D, Swann C, Allen MS, \& Vella SA (2020). A qualitative evaluation of a mental health literacy intervention for parents delivered through community sport clubs. Psychology of Sport and Exercise 47: 101635

35. Bapat S, Jorm A, Lawrence K (2009). Evaluation of a mental health literacy training program for junior sporting clubs. Australasian Psychiatry 17:475-479.

36. Pierce D, Liaw ST, Dobell J , \& Anderson, RJ (2010). Australian rural football club leaders as mental health advocates: An investigation of the influence of the Coach the Coach project. International Journal of Mental Health Systems 4:10-17.

37. Australian Bureau of Statistics (2008). Information paper. An introduction to SocioEconomic Indexes for Areas (SEIFA) 2006. Catalogue no. 2039.0. Canberra: ABS.

38. Griffiths KM, Christensen H, Jorm AF, Evans K, Groves C (2004). Effect of webbased depression literacy and cognitive-behavioural therapy interventions on stigmatising attitudes to depression. The British Journal of Psychiatry 185:342-349.

39. O’ Connor M, Casey L (2015) The Mental Health Literacy Scale (MHLS): A new scale based measure of mental health literacy. Psychiatry Research 229:111-516.

40. Wilson CJ, Deane FP, Ciarrochi J Rickwood D (2005). Measuring help- seeking intentions: Properties of the General Help-Seeking Questionnaire. Canadian Journal of Counselling 39:15-28.

41. Kessler RC, Andrews G, Colpe LJ, Hiripi E, Mroczek DK, Normand SL, ... Zaslavsky AM (2002). Short screening scales to monitor population prevalences and trends in non-specific psychological distress. Psychological Medicine 32:959-976. 
42. Vella SA, Swann C, Batterham M, Boydell, KM, Eckermann S, Fogarty A ... Noetel M (2018). Ahead of the game protocol: A multi-component, community sport-based program targeting prevention, promotion and early intervention for mental health among adolescent males. BMC Public Health 18:390-401.

43. Minkler M, Wallerstein N (2003). Community-based participatory research for health. San Francisco, CA, Jossey-Bass.

44. Ingoldsby EM (2010). Review of interventions to improve family engagement and retention in parent and child mental health programs. Journal of Child and Family Studies 19:629-645.

45. Fischer JA, Kelly CM, Kitchener BA, Jorm AF (2013). Development of guidelines for adults on how to communicate with adolescents about mental health problems and other sensitive topics. SAGE Open 3:1-15.

46. Morgan AJ, Jorm AF (2009). Self-help strategies that are helpful for sub-threshold depression: A Delphi consensus study. Journal of Affective Disorders 115:196-200.

47. Cutler TL, Reavley NJ, \& Jorm AF (2017). How 'mental health smart'are you? Analysis of responses to an Australian Broadcasting Corporation News website quiz. Advances in Mental Health 16:5-18.

48. Gibbons RD, Hedeker D, Du Toit S (2010). Advances in analysis of longitudinal data. Annual Review of Clinical Psychology 6:79-107.

49. Becker H, Roberts G, Voelmeck W (2003). Explanations for improvement in both experimental and control groups. Western Journal of Nursing Research 25:746-755.

50. Thurston IB, Phares V, Coates EE, Bogart LM (2015). Child problem recognition and help-seeking intentions among black and white parents. Journal of Clinical Child Adolescent Psychology 44:604-615. 
51. Cairns K, Potter S, Nicholas M, Buhagiar K (2019). Development of ReachOut Parents: A multi-component online program targeting parents to improve youth mental health outcomes. Advances in Mental Health 17:55-71.

52. Poole LA, Knight T, Toumbourou JW, Lubman DI, Bertino MD, Lewis AJ (2018). A randomized controlled trial of the impact of a family-based adolescent depression intervention on both youth and parent mental health outcomes. Journal of Abnormal Child Psychology 46:169-181.

53. Brijnath B, Protheroe J, Mahtani KR, Antoniades J (2016). Do web-based mental health literacy interventions improve the mental health literacy of adult consumers? Results from a systematic review. Journal of Medical Internet Research 18:e165.

54. Goldney, RD, Eckert KA, Hawthorne G, Taylor AW (2010). Changes in the prevalence of major depression in an Australian community sample between 1998 and 2008. Australian New Zealand Journal of Psychiatry 44:901-910.

55. Baker CN, Arnold DH, Meagher S (2011). Enrollment and attendance in a parent training prevention program for conduct problems. Prevention Science 12:126-138.

56. Jung H, von Sternberg K, Davis K (2017). The impact of mental health literacy, stigma, and social support on attitudes toward mental health help-seeking. International Journal of Mental Health Promotion 19:252-267.

57. Vella, SA, Cliff DP, \& Okely AD (2014). Socio-ecological predictors of participation and dropout in organised sports during childhood. International Journal of Behavioral Nutrition and Physical Activity 11: 62-81.

58. Morgan AJ, Ross A, Reavley NJ (2018). Systematic review and meta-analysis of Mental Health First Aid training: Effects on knowledge, stigma, and helping behaviour. PloS One 13:e197102. 
710

711

712

713

59. Bertoni, A., Donato, S., Morgano, A., Iafrate, R., \& Rosnati, R. (2017). A qualitative evaluation of a preventive intervention for parents: The Groups for Family Enrichment_Parent version (GFE_P). Journal of Prevention \& Intervention in the Community, 45, 215-229. 
Table 1

Intervention Workshop Outline

\begin{tabular}{|c|c|c|c|}
\hline $\begin{array}{l}\text { Mental Health Literacy } \\
\text { Component }\end{array}$ & Objective/s & Activities and resources & Time allotted \\
\hline $\begin{array}{l}\text { Attitudes towards seeking } \\
\text { knowledge and help }\end{array}$ & $\begin{array}{l}\text { Raise awareness of parents' role in } \\
\text { supporting positive adolescent } \\
\text { mental health }\end{array}$ & $\begin{array}{l}\text { Facilitator led group discussion } \\
\text { ReachOut Video: Adolescents need parent support }\end{array}$ & $\sim 10$ mins \\
\hline $\begin{array}{l}\text { Capacity to recognize the } \\
\text { development or signs of a } \\
\text { disorder }\end{array}$ & $\begin{array}{l}\text { Learn about Depression and } \\
\text { Anxiety and how to differentiate } \\
\text { symptoms from normal teenage } \\
\text { behaviour }\end{array}$ & $\begin{array}{l}\text { Discussion on regular teenage behaviour } \\
\text { Information on symptoms and when to take action } \\
\text { Scenarios: What would you do? }\end{array}$ & $\sim 20 \mathrm{mins}$ \\
\hline $\begin{array}{l}\text { Knowledge about } \\
\text { professional help-seeking } \\
\text { and treatment options }\end{array}$ & $\begin{array}{l}\text { Raise awareness and knowledge of } \\
\text { professional help seeking services } \\
\text { available to parents and youth }\end{array}$ & $\begin{array}{l}\text { Information and discussion of professional help-seeking options } \\
\text { ReachOut Video: What to expect when seeing a psychologist }\end{array}$ & $\sim 15$ mins \\
\hline Capacity to help & $\begin{array}{l}\text { Learn how to communicate about } \\
\text { mental health with teenagers }\end{array}$ & $\begin{array}{l}\text { Step by step guide to communicating about mental health with } \\
\text { adolescents } \\
\text { (Fischer, Kelly, Kitchener Jorm, 2013). } \\
\text { Video "Talking about teenage depression" Raising Children } \\
\text { Network website } \\
\text { When someone doesn't want help }\end{array}$ & $\sim 15$ mins \\
\hline $\begin{array}{l}\text { Knowledge of preventive } \\
\text { and self-help strategies }\end{array}$ & $\begin{array}{l}\text { Explore ways parents can } \\
\text { encourage mentally healthy } \\
\text { behaviours in their children }\end{array}$ & $\begin{array}{l}\text { Group discussion and outline of mentally healthy strategies } \\
\text { (Morgan Jorm, 2009; Yap et al., 2014) }\end{array}$ & $\sim 5$ mins \\
\hline $\begin{array}{l}\text { Knowledge and capacity to } \\
\text { help }\end{array}$ & $\begin{array}{l}\text { Raise awareness and knowledge of } \\
\text { mental health resources available to } \\
\text { parents }\end{array}$ & $\begin{array}{l}\text { Handout pamphlet with key information from workshop and list } \\
\text { of mental health organisations and websites. } \\
\text { Access to optional supplementary online material }\end{array}$ & Post workshop \\
\hline
\end{tabular}


Table 2

Mean scores and standard errors for intervention and control groups at baseline and one month follow-up

\begin{tabular}{|c|c|c|c|c|c|c|c|c|c|}
\hline & \multicolumn{4}{|c|}{ Intervention Group } & \multicolumn{4}{|c|}{ Control Group } & \multirow[b]{3}{*}{$\eta_{\mathrm{p}}^{2}($ partial eta squared) } \\
\hline & \multicolumn{2}{|c|}{ Baseline } & \multicolumn{2}{|c|}{ Follow-up } & \multicolumn{2}{|c|}{ Baseline } & \multicolumn{2}{|c|}{ Follow-up } & \\
\hline & M & SE & M & SE & M & SE & M & SE & \\
\hline Depression literacy & 8.83 & .21 & 9.89 & .24 & 8.52 & .29 & 9.02 & .40 & .002 \\
\hline Anxiety literacy & 7.36 & .24 & 8.49 & .26 & 6.86 & .31 & 7.41 & .43 & .006 \\
\hline Confidence and knowledge & 8.06 & .10 & 8.63 & .11 & 8.38 & .13 & 8.25 & .19 & $.034 * *$ \\
\hline Stigmatizing attitudes & 18.71 & .32 & 19.54 & .33 & 19.10 & .41 & 19.47 & .53 & .003 \\
\hline Attitudes that promote help-seeking & 38.77 & .43 & 38.98 & .46 & 38.60 & .54 & 37.78 & .73 & .007 \\
\hline Personal informal help-seeking & 4.84 & .10 & 4.80 & .11 & 4.93 & .13 & 4.83 & .18 & .000 \\
\hline Personal formal help-seeking & 4.46 & .12 & 4.99 & .13 & 4.51 & .15 & 4.60 & .20 & $.016^{*}$ \\
\hline Informal help-seeking for child & 5.15 & .10 & 4.25 & .11 & 5.24 & .13 & 4.24 & .19 & .000 \\
\hline Formal help-seeking for child & 5.14 & .11 & 5.15 & .12 & 5.24 & .20 & 5.47 & .29 & .004 \\
\hline Confidence & 3.19 & .09 & 3.43 & .09 & 3.26 & .96 & 3.19 & .96 & $.011^{*}$ \\
\hline Parent support & 6.93 & .19 & 7.06 & .19 & 7.08 & .23 & 6.63 & .30 & $.013^{*}$ \\
\hline Psychological distress & 11.65 & .28 & 11.17 & .30 & 11.15 & .35 & 11.71 & .47 & $.015^{*}$ \\
\hline
\end{tabular}

${ }^{*} p<.05, * * p<.001$ 
Table 3

Unadjusted mean scores, standard deviations and effect size differences for outcome measures, parent sex, previous contact, and mental health disorder diagnosis

\begin{tabular}{|c|c|c|c|c|c|c|c|c|c|}
\hline & \multicolumn{3}{|l|}{ Sex } & \multicolumn{3}{|c|}{ Previous Contact } & \multicolumn{3}{|c|}{ Mental Health Disorder Diagnosis } \\
\hline & Men & Women & & Yes & No & & Yes & No & \\
\hline & $\mathrm{M}(\mathrm{SD})$ & $\mathrm{M}(\mathrm{SD})$ & $d$ & $\mathrm{M}(\mathrm{SD})$ & $\mathrm{M}(\mathrm{SD})$ & $d$ & $\mathrm{M}(\mathrm{SD})$ & $\mathrm{M}(\mathrm{SD})$ & $d$ \\
\hline Depression literacy & $8.79(2.76)$ & $9.56(2.53)$ & $0.29 *$ & $9.87(2.29)$ & $8.76(2.82)$ & $0.43 * *$ & $9.55(2.29)$ & $9.22(2.69)$ & 0.13 \\
\hline Anxiety literacy & $7.23(2.92)$ & $8.32(2.76)$ & $0.38 * *$ & $8.44(2.63)$ & $7.45(2.98)$ & $0.35^{* *}$ & $8.10(2.54)$ & $7.86(2.91)$ & 0.08 \\
\hline Confidence and knowledge & $7.90(1.20)$ & $8.50(1.27)$ & $0.48 * *$ & $8.57(1.19)$ & $8.02(1.29)$ & $0.44 * *$ & $8.47(1.14)$ & $8.24(1.29)$ & 0.18 \\
\hline Stigmatizing attitudes & $18.00(3.62)$ & $19.75(3.82)$ & $0.47 * *$ & $20.18(3.76)$ & $18.22(3.68)$ & $0.53 * *$ & $20.04(4.17)$ & $18.99(3.80)$ & 0.26 \\
\hline Attitudes that promote help-seeking & $37.86(4.76)$ & $39.95(4.46)$ & $0.45^{* *}$ & $40.15(4.12)$ & $38.30(4.95)$ & $0.41^{* *}$ & $39.15(5.11)$ & $39.11(4.65)$ & 0.01 \\
\hline Personal informal help-seeking & $4.79(1.08)$ & $5.04(1.23)$ & 0.21 & $4.98(1.13)$ & $4.90(1.23)$ & 0.07 & $4.80(1.27)$ & $4.95(1.16)$ & 0.12 \\
\hline Personal formal help-seeking & $4.65(1.28)$ & $4.72(1.29)$ & 0.05 & $4.78(1.26)$ & $4.61(1.30)$ & 0.12 & $4.53(1.42)$ & $4.71(1.28)$ & 0.13 \\
\hline Informal help-seeking for child & $4.91(1.09)$ & $4.81(1.24)$ & 0.08 & $4.84(1.15)$ & $4.85(1.22)$ & 0.02 & $4.76(1.26)$ & $4.87(1.17)$ & 0.10 \\
\hline Formal help-seeking for child & $5.18(1.08)$ & $5.21(1.19)$ & 0.03 & $5.24(1.11)$ & $5.17(1.17)$ & 0.06 & $5.19(1.11)$ & $5.20(1.14)$ & 0.01 \\
\hline Confidence & $3.14(1.02)$ & $3.34(.98)$ & 0.20 & $3.44(.98)$ & $3.11(1.01)$ & $0.33^{*}$ & $3.27(.98)$ & $3.26(1.01)$ & 0.01 \\
\hline Psychological distress & $9.49(3.09)$ & $9.93(3.30)$ & 0.14 & $10.18(3.57)$ & $9.42(2.87)$ & 0.24 & $13.41(4.75)$ & $9.37(2.74)$ & $1.04 * *$ \\
\hline Parent support & $6.74(1.76)$ & $7.18(1.91)$ & 0.24 & $7.05(1.85)$ & $6.97(1.87)$ & 0.04 & $6.88(1.79)$ & $7.02(1.87)$ & 0.07 \\
\hline
\end{tabular}

Note. Previous contact refers to previous recent contact with someone who had a mental health disorder. Mental health disorder diagnosis refers to any personal experience of having had a mental health disorder.

Depression and Anxiety Literacy scales are scored 0-13 with higher scores indicating better disorder specific knowledge. The Confidence and Knowledge subscale and Parent Support scales are scored 2-10 with higher scores indicating more confidence and knowledge to seek help, and more perceived support from other parents respectively. Stigmatising attitudes subscale is scored 5-25 with higher scores indicating less stigmatizing attitudes. Attitudes subscale is scored 9-45 with higher scores indicating more favorable attitudes towards mental health disorders and help-seeking. Help seeking scales are scored 1-7 with higher scores indicating greater help-seeking intentions. Psychological Distress is scored 6-30 with higher scores indicating more psychological distress.

$* p<.01, * * p<.00$ 
Table 4

Moderator analysis of parent sex, previous contact, and mental health disorder diagnosis for significant group x time interactions

\begin{tabular}{|c|c|c|c|c|c|c|c|c|c|}
\hline & \multicolumn{3}{|l|}{ Sex } & \multicolumn{3}{|c|}{ Previous Contact } & \multicolumn{3}{|c|}{ Mental Health Disorder Diagnosis } \\
\hline & $F$ & $d f$ & $p$ & $F$ & $d f$ & $p$ & $F$ & $d f$ & $p$ \\
\hline Confidence and knowledge & 1.41 & $1,253.34$ & .237 & 3.074 & $1,248.10$ & .081 & 1.995 & $1,304.10$ & .159 \\
\hline Personal formal help-seeking & .253 & $1,246.80$ & .615 & .949 & $1,242.04$ & .331 & .070 & $1,292.41$ & .791 \\
\hline Confidence & .230 & 1.249 .89 & .130 & .029 & $1,247, .21$ & .866 & .176 & $1,339.68$ & .675 \\
\hline Psychological distress & .612 & $1,245.64$ & .435 & .600 & $1,242.99$ & .430 & 5.28 & $1,304.77$ & $.022 *$ \\
\hline Parent support & .004 & $1,247.60$ & .947 & 1.759 & $1,241.72$ & .186 & .405 & $1,326.16$ & .525 \\
\hline
\end{tabular}

